\title{
Die Anwendung statistischer Methoden in der Markt- und Meinungsforschung in Österreich
}

\author{
Udo Wagner, Heribert Reisinger \\ Institut für Betriebswirtschaftslehre, Universität Wien
}

\begin{abstract}
Zusammenfassung: Der rasanten Entwicklung des statistischen Instrumentariums in der akademischen Forschung steht eine erhebliche Diskrepanz bei der Anwendung dieser Methoden in der Praxis von österreichischen Markt- und Meinungsforschungsinstituten gegenüber. Der vorliegende Beitrag möchte dies einerseits nachweisen; andererseits wird an Hand konkreter Beispiele aufgezeigt, daß statistische Untersuchungen wertvolle Erkenntnisse für die betriebliche Entscheidungsfindung liefern können. Das oben angesprochene Mißverhältnis dürfte auf Gründe wie etwa eine generelle Reserviertheit der potentiellen Anwender bedingt durch negative Erfahrungen im Rahmen der bisherigen Tätigkeit oder der Ausbildung zurückzuführen sein. Schließlich werden einige Vorschläge zur Verbesserung der gegenwärtigen Situation unterbreitet.
\end{abstract}

\begin{abstract}
In spite of the progress of academic research in the area of statistics, the application of these methods within the commercial Austrian opinion and marketing research industry considerably lags behind. The paper at hand investigates this issue; furthermore, a number of real world examples are provided which demonstrate the usefulness of statistical methods in order to yield additional insights assisting management decision making. The discrepancy mentioned above is probably due to a rather general negative attitude of potential clients because of their bad experiences in the course of previous activities or during their education at university. Finally, recommendations for improving the current situation are given.
\end{abstract}

Schlüsselwörter: Statistik, Markt- und Meinungsforschung, empirische Regelmäßigkeit im Marketing, Metaanalyse, Fallbeispiele.

\section{Problemstellung}

Im Rahmen unserer Tätigkeit in Forschung und Lehre sowie bei der Durchführung von Projekten in Zusammenarbeit mit österreichischen Unternehmen befassen wir uns häufig mit der Analyse von empirischen Daten über das Kaufverhalten oder die Zufriedenheit von Konsumenten und über die Entwicklung von Marktanteilen in Abhängigkeit von verschiedenen Marketing-Mix Instrumenten im Zeitablauf. Typischerweise werden dabei Methoden der Statistik verwendet, mit deren Hilfe zumeist interessante Erkenntnisse gewonnen werden können. Diesem positiven Aspekt steht oft die Beobachtung gegenüber, daß die Akzeptanz dieses Instrumentariums bei vielen unserer Kooperationspartner auBerordentlich gering ist. Dies gilt einerseits für Studierende betriebswirtschaftlicher Studienrichtungen, die vielfach mit geringem Wissen und noch bescheidenerem Verständnis 
sowie mangelhafter Motivation statistische Verfahren anwenden (müssen). Andererseits sind wir aber auch in der Praxis sehr oft mit Persönlichkeiten konfrontiert, deren Einstellung zur Statistik meist zwischen Skepsis oder bewußt artikuliertem Unverständnis (im Sinne einer fehlenden Bereitschaft, sich mit solchen Sachverhalten auseinanderzusetzen) schwankt; im positiven Fall begegnet uns Bewunderung für die Beherrschung einer "akademischen Fertigkeit", die ihrer Meinung nach ausschließlich Spezialisten vorbehalten bleibt.

Vor diesem Hintergrund und der Tatsache, daß die Österreichische Zeitschrift für Statistik ein Publikum anspricht, welches der statistischen Forschung positiv gegenüber steht, wollen wir mit dem vorliegenden Beitrag ein dreifaches Ziel erreichen:

1. Bewußtmachen der Diskrepanz zwischen dem "state-of-the-art" in Statistik und ihrer Anwendung in kommerziellen österreichischen Markt- und Meinungsforschungsinstituten (Abschnitt 2);

2. Aufzeigen typischer Einsatzmöglichkeiten für statistische Methoden zur Gewinnung neuer Erkenntnisse im Marketing (Abschnitt 3);

3. Demonstrieren der Aussagekraft einfacher statistischer Verfahren zur Unterstützung der betrieblichen Entscheidungsfindung an Hand eines realen Fallbeispiels (Abschnitt 4).

Es geht uns also um die Verbesserung der Kommunikation zwischen Entwicklern und betriebswirtschaftlichen Anwendern des statistischen Instrumentariums, und es werden schließlich potentielle gemeinsame Arbeitsschwerpunkte aufgezeigt (Abschnitt 5).

\section{Ergebnisse einer Befragung}

Das oben angesprochene Mißverhältnis war uns auf Grund unserer persönlichen Erfahrungen zwar durchaus bewußt, wir wollten es aber in diesem Aufsatz anhand besser belegbarer Argumente aufzeigen. Deshalb befragten wir im März 1997 die kommerziellen österreichischen Markt- und Meinungsforschungsinstitute telephonisch zu diesem Thema. Wir wählten dabei alle jene Unternehmen subjektiv aus, die in der Branche dafür bekannt sind, auch "statistische" Auswertungen durchzuführen. Als Gesprächspartner dieser explorativen Untersuchung fungierte jeweils der Leiter der Methodenabteilung. Zunächst wurde er gefragt, “... welche statistischen Kennzahlen, Methoden und graphischen Darstellungen ..." verwendet werden, wobei bei jeder Nennung nachgefragt wurde, ob der Einsatz der angegebenen Methode häufig $(\mathrm{H})$, mittel $(\mathrm{M})$ oder selten $(\mathrm{S})$ erfolgt. Die erhaltenen Antworten sind in Tabelle 1 unter der Spalte "Verwendung ungestützt" wiedergegeben; das " $\mathrm{H}$ " in der Zeile "arithmetisches Mittel" ist der Median und bedeutet daher, daß mehr als die Hälfte der 15 erfaßten Institute häufig arithmetische Mittelwerte berechnen. Diese Aussage gilt auch für die Verwendung von Graphiken, wobei insbesondere Kreis- und Balkendiagramme sowie Häufigkeitsverteilungen genannt wurden. Alle anderen Methoden wurden nur vereinzelt angegeben! 
Im zweiten Teil des Interviews wurde nun für alle in Tabelle 1 aufgezählten Methoden direkt abgefragt, ob beispielsweise Regressionsanalysen durchgeführt werden. Auch hier sind die Ergebnisse aus Tabelle 1 ersichtlich (Verwendung "gestützt"), wobei zusätzlich die Antworten je Klasse angegeben werden. Die Tendenz ist eindeutig erkennbar: eigentlich werden nur beschreibende Verfahren regelmäßig eingesetzt, univariate Tests von einigen Instituten, multivariate Verfahren eher selten. Es ist festzuhalten, daß die angeführten Methoden zum statistischen Standard gehören, in einschlägigen Softwarepaketen enthalten sind und zumeist im Rahmen der Grundausbildung an Universitäten gelehrt werden. Vom Standpunkt des Statistikers sind diese Resultate daher ernüchternd, insbesondere wenn man berücksichtigt, daß Unternehmen interviewt wurden, die professionell Datenanalysen anbieten und hier wiederum solche, die eher methodenorientiert arbeiten als die Nichtbefragten.

Tabelle 1: Verwendung statistischer Methoden durch österreichische Markt- und Meinungsforschungsinstitute

\begin{tabular}{|l||c|c|c|c|c|c|}
\hline \multicolumn{1}{|c||}{} & Verwendung & Verwendung & \multicolumn{3}{|c|}{ Verwendung gestützt } \\
Methode & $\begin{array}{c}\text { - Median } \\
\text { gestützt }\end{array}$ & $\begin{array}{c}\mathrm{H} \\
\text { - Median }\end{array}$ & $\begin{array}{c}\text { M } \\
\text { häufig }\end{array}$ & $\begin{array}{c}\text { S } \\
\text { mittel }\end{array}$ & Nelten & nie \\
\hline \hline Beschreibende Statistiken & & & & & & \\
\hline - arithmetisches Mittel & $\mathrm{H}$ & $\mathrm{H}$ & 13 & & 2 & \\
- Median & $\mathrm{N}$ & $\mathrm{S}$ & 4 & 2 & 7 & 2 \\
- Varianz / Standardabw. & $\mathrm{N}$ & $\mathrm{H}$ & 8 & 2 & 4 & 1 \\
- Quartile & $\mathrm{N}$ & $\mathrm{S}$ & 2 & 3 & 7 & 3 \\
- Häufigkeitsverteilungen & $\mathrm{N}$ & $\mathrm{H}$ & 11 & & 2 & 2 \\
- Kreuztabellen & $\mathrm{N}$ & $\mathrm{H}$ & 12 & & 2 & 1 \\
- Graphiken & $\mathrm{H}$ & $\mathrm{H}$ & 14 & & 1 & \\
\hline Tests & & & & & & \\
\hline - t-Test & $\mathrm{N}$ & $\mathrm{M}$ & 7 & 1 & 5 & 2 \\
- F-Test & $\mathrm{N}$ & $\mathrm{S}$ & 4 & & 6 & 5 \\
- Chi-Quadrat-Test & $\mathrm{N}$ & $\mathrm{M}$ & 7 & 1 & 6 & 1 \\
\hline Multivariate Verfahren & & & & & & \\
\hline - Regressionsanalyse & $\mathrm{N}$ & $\mathrm{M}$ & 4 & 4 & 4 & 3 \\
- Varianzanalyse & $\mathrm{N}$ & $\mathrm{S}$ & 2 & 3 & 7 & 3 \\
- Diskriminanzanalyse & $\mathrm{N}$ & $\mathrm{S}$ & 2 & 2 & 6 & 5 \\
- Faktorenanalyse & $\mathrm{N}$ & $\mathrm{S}$ & 5 & 2 & 7 & 1 \\
- Clusteranalyse & $\mathrm{S}$ & $\mathrm{M}$ & 6 & 3 & 6 & \\
- Multidimensionale Skalierung & $\mathrm{N}$ & $\mathrm{S}$ & 2 & 1 & 11 & 1 \\
- Conjoint-Analyse & $\mathrm{N}$ & $\mathrm{S}$ & 2 & 2 & 6 & 5 \\
- Zeitreihenanalyse & $\mathrm{N}$ & $\mathrm{S}$ & 6 & & 4 & 5 \\
\hline
\end{tabular}

Obwohl wir die Gründe für diese Sachlage nicht empirisch untersucht haben, liegt eine Erklärung dafür auf der Hand, nämlich mangelnde Nachfrage seitens der Auftraggeber von Marktforschungsinstituten. Diese haben möglicherweise schlechte Erfahrungen im Umgang mit Statistik im Rahmen ihrer Ausbildung oder ihrer bisherigen beruflichen Laufbahn gemacht, oder ihre Meinung über die Verläßlichkeit von statistischen Aussagen ist etwa auf Grund von widersprüchlichen Hochrechnungen im Zuge politischer 
Meinungsforschung negativ geprägt. Es ist wohl auch eine Tatsache, daß Statistiken oft (bewußt) mißinterpretiert werden (siehe in diesem Zusammenhang etwa SACHS (1997), KRÄMER (1995), DEWDNEY (1994)).

\section{Erkenntnisgewinn im Marketing}

Gesicherte, möglichst allgemeingültige Erkenntnisse im Marketing gibt es zur Zeit noch relativ wenige. Das mag einerseits daran liegen, daß Marketing als Teildisziplin der Sozial- und Wirtschaftswissenschaften mit all den bekannten Schwierigkeiten zu kämpfen hat, die aus der Mannigfaltigkeit menschlichen Handelns resultieren, andererseits aber auch daran, daß es sich um ein relativ junges Fachgebiet handelt. Einige Forschungsanstrengungen sind zur Zeit daher von der Überlegung geleitet, daß durch die Untersuchung einer Vielzahl von Einzelfällen globale Aussagen ableitbar sind. Dies vollzieht sich zum einen auf der Ebene konkreter Analyseobjekte (beispielsweise empirisch beobachteter Märkte über bestimmte Produkte in bestimmten Zeiträumen und Regionen) und zum anderen auf einem höheren Abstraktionsniveau, auf dem nur mehr bereits komprimiertes Informationsmaterial vorliegt. In beiden Fällen liefert jedoch die Statistik das verwendete Analyseinstrumentarium. Die folgenden beiden Unterabschnitte geben dafür jeweils ein Beispiel und sollen so typische Einsatzmöglichkeiten für statistische Werkzeuge im Marketing charakterisieren.

\subsection{Empirisch beobachtete Regelmäßigkeiten beim Kaufverhalten von Konsumenten}

Die folgenden Ausführungen orientieren sich stark an EHRENBERG (1988) und beziehen sich auf regelmäßig nachgefragte Konsumgüter.

Das Kaufverhalten ist ein komplexer Vorgang. Jede Kaufentscheidung wird sowohl von endogenen (in der Person des Käufers liegenden) als auch exogenen Faktoren beeinflußt. Endogene Faktoren sind z. B. psychologische Einflüsse und soziodemographische Charakteristika, während sich die kaufrelevanten exogenen Faktoren u. a. aus dem Marketing-Mix, allfälligen Erfahrungen mit dem Produkt und Mund-zu-Mund Effekten zusammensetzen. Die Kaufentscheidung selbst beinhaltet die vier Elemente Einkaufszeitpunktswahl, Markenwahl, Ladenwahl und Mengenwahl.

Ein möglicher Zugang bei der Analyse des Kaufverhaltens von Konsumenten besteht darin, von den beobachteten Kaufakten eines Haushalts (in Form von Panel- bzw. Scannerdaten) auszugehen und alle potentiellen Einflußfaktoren zunächst auszublenden. Ist man insbesondere am Wiederkaufverhalten von Konsumenten interessiert, so ist es möglich, bestimmte Regelmäßigkeiten zu erkennen, die unten beschrieben und anhand eines Beispiels illustriert werden. Folgende beobachtbare Größen sind bei der Analyse des Wiederkaufverhaltens von zentraler Bedeutung (wobei von zwei gleich langen, aufeinanderfolgenden Zeitintervallen ausgegangen wird; die angegebenen Abkürzungen beziehen sich auf die Bezeichnung der entsprechenden Spalten in Tabelle 2): 
MitTlere KaUfrate (mittl. Kaufr.):

durchschnittliche Anzahl von Einkäufen je Käufer im Beobachtungszeitraum;

ABSATZ WIEDERKÄUFER (Absatz Wied.k.):

Absatz, der jenen Konsumenten zuzuordnen ist, die sowohl im Beobachtungsintervall als auch im vorhergehenden Intervall gekauft haben (in Prozent des Gesamtabsatzes);

AbSATZ KAUfintensiver NACHFRAGER (Absatz mind.2x, 3x, 4x, 6x):

Absatz, der jenen Konsumenten zuzuordnen ist, die im Beobachtungszeitraum zumindest zweimal, dreimal, viermal, ... gekauft haben (in Prozent des Gesamtabsatzes);

KAUFRATE VON ERSTKÄUFERN (Kaufr. Erstk.):

durchschnittliche Anzahl von Einkäufen je Erstkäufer (das sind jene, die im vorhergehenden Intervall nicht gekauft haben).

Tabelle 2: Regelmäßigkeiten beim Kaufverhalten

\begin{tabular}{|l||c|c|c|c|c|c|c|c|}
\hline $\begin{array}{l}\text { Marke (Pro- } \\
\text { duktkategorie) }\end{array}$ & $\begin{array}{c}\text { mittl. } \\
\text { Kaufr. }\end{array}$ & $\begin{array}{c}\text { Absatz } \\
\text { Wied.k. }\end{array}$ & $\begin{array}{c}\text { Absatz } \\
\text { mind.2x }\end{array}$ & $\begin{array}{c}\text { Absatz } \\
\text { mind.3x }\end{array}$ & $\begin{array}{c}\text { Absatz } \\
\text { mind.4x }\end{array}$ & $\begin{array}{c}\text { Absatz } \\
\text { mind.6x }\end{array}$ & $\begin{array}{c}\text { Kaufr. } \\
\text { Erstk. }\end{array}$ & $\begin{array}{c}\text { Beob. } \\
\text { int. }\end{array}$ \\
\hline \hline $\begin{array}{l}\text { Palmolive } \\
\text { (Seife) }\end{array}$ & 1.31 & $33 \%$ & $41 \%$ & $17 \%$ & $6 \%$ & 0 & 1.22 & $6 \mathrm{M}$ \\
\hline $\begin{array}{l}\text { Pril (Geschirr- } \\
\text { spülmittel) }\end{array}$ & 1.37 & $42 \%$ & $41 \%$ & $28 \%$ & $12 \%$ & $7 \%$ & 1.23 & $6 \mathrm{M}$ \\
\hline $\begin{array}{l}\text { Ariel (Univer- } \\
\text { salwaschmittel) }\end{array}$ & 1.62 & $54 \%$ & $59 \%$ & $35 \%$ & $17 \%$ & $9 \%$ & 1.29 & $6 \mathrm{M}$ \\
\hline $\begin{array}{l}\text { Mentadent } \\
\text { (Zahncreme) }\end{array}$ & 1.75 & $58 \%$ & $63 \%$ & $43 \%$ & $29 \%$ & $15 \%$ & 1.27 & $6 \mathrm{M}$ \\
\hline $\begin{array}{l}\text { Teekanne Kräu- } \\
\text { tertee (Tee) }\end{array}$ & 1.81 & $61 \%$ & $65 \%$ & $46 \%$ & $31 \%$ & $11 \%$ & 1.30 & $4 \mathrm{M}$ \\
\hline $\begin{array}{l}\text { Milfina } \\
\text { (Schmelzkäse) }\end{array}$ & 1.93 & $67 \%$ & $70 \%$ & $49 \%$ & $36 \%$ & $14 \%$ & 1.30 & $4 \mathrm{M}$ \\
\hline $\begin{array}{l}\text { Carnis Rind- } \\
\text { suppe (Suppe) }\end{array}$ & 2.00 & $73 \%$ & $69 \%$ & $51 \%$ & $41 \%$ & $22 \%$ & 1.22 & $14 \mathrm{~W}$ \\
\hline $\begin{array}{l}\text { Gold Extra } \\
\text { (Bohnenkaffee) }\end{array}$ & 2.16 & $77 \%$ & $77 \%$ & $53 \%$ & $42 \%$ & $18 \%$ & 1.37 & $9 \mathrm{~W}$ \\
\hline $\begin{array}{l}\text { Vita } \\
\text { (Margarine) }\end{array}$ & 2.31 & $82 \%$ & $80 \%$ & $64 \%$ & $44 \%$ & $17 \%$ & 1.36 & $6 \mathrm{~W}$ \\
\hline $\begin{array}{l}\text { Coca Cola } 1,51 \\
\text { (Limonade) }\end{array}$ & 2.44 & $73 \%$ & $80 \%$ & $64 \%$ & $51 \%$ & $31 \%$ & 1.54 & $7 \mathrm{~W}$ \\
\hline $\begin{array}{l}\text { Wieselburger } \\
\text { Gold (Bier) }\end{array}$ & 3.10 & $81 \%$ & $86 \%$ & $74 \%$ & $61 \%$ & $47 \%$ & 1.35 & $9 \mathrm{~W}$ \\
\hline
\end{tabular}

Tabelle 2 präsentiert diese Größen für ausgewählte Marken aus verschiedenen Produktkategorien (die zugehörigen Ausgangsdaten stammen aus dem österreichischen GfKHaushaltspanel für das Jahr 1992). Die Marken sind nach der mittleren Kaufrate in ansteigender Weise angeordnet. Die Länge des jeweiligen Beobachtungsintervalls (Beob.int.) 
wurde in Anlehnung an BASS, LEONE (1983) ermittelt, indem der Wert 52 (stellvertretend für die Anzahl der Wochen eines Jahres) durch die jahresbezogene mittlere Kaufrate dividiert wurde. Betrug der berechnete Zeitraum über 15 Wochen, so wurde auf ganze Monate gerundet. Die Abkürzungen "M" und "W" stehen für "Monate" bzw. "Wochen", das bedeutet beispielsweise, daß Palmolive (Seife) im Jahresdurchschnitt von allen Käufern dieser Marke ungefähr zweimal nachgefragt worden ist. Diese Transformation erleichtert die simultane Betrachtung von Produkten unterschiedlicher Kaufhäufigkeiten.

Wie aus Tabelle 2 ersichtlich steigt mit der mittleren Kaufrate auch der Absatz der Wiederkäufer und der Absatz kaufintensiver Nachfrager allmählich an. Die Kaufrate der Erstkäufer bleibt hingegen annähernd konstant. Weiters ist zu erkennen, daß der Absatz der Wiederkäufer und der Absatz jener Konsumenten, die zumindest zweimal gekauft haben, ungefähr gleich groß ist. Folgende, durchgängig zu beobachtenden Phänomene lassen sich damit aus Tabelle 2 ableiten:

- Mit der mittleren Kaufrate schwankt auch der Absatz der Wiederkäufer.

- In ähnlicher Weise schwankt auch der Absatz der Käufer, die zumindest zweimal gekauft haben.

- Der Absatz der Wiederkäufer und jener, die zumindest zweimal gekauft haben, ist nahezu gleich.

- Auch der Absatz kaufintensiver Nachfrager schwankt mit der mittleren Kaufrate.

- Die Kaufrate der Erstkäufer ist beinahe konstant.

In Tabelle 2 wurden verschiedene Marken aus verschiedenen Produktkategorien aufgelistet. Die beobachteten Regelmäßigkeiten gelten jedoch auch für verschiedene Marken innerhalb einer Produktkategorie, für verschiedene Produktkategorien auf aggregiertem Niveau, für verschiedene Packungsgrößen und im internationalen Vergleich. Voraussetzung bei der Ableitung der Phänomene ist lediglich das Vorliegen von Stationarität, d. h., daß das Absatzvolumen der untersuchten Marken in den Beobachtungsintervallen annähernd konstant sein sollte. Abweichungen vom erwarteten Verlauf (siehe beispielsweise den Absatz der Wiederkäufer von Coca Cola in der 1,5 1 Flasche) können daher zum Teil durch das Vorliegen von nicht-stationären Situationen erklärt werden.

Einfache Formeln wurden entwickelt, um die beschriebenen Regelmäßigkeiten zu modellieren (siehe EHRENBERG, 1988, S. 21 ff.), die ihrerseits wiederum als Ausgangsbasis für die Entwicklung des LSD (logarithmic series distribution)- bzw. NBD (negative binomial distribution)-Modells - zwei bekannte stochastische Kaufverhaltensmodelle dienten. Bezüglich einer allgemeinen Einführung in die Klasse der stochastischen Kaufverhaltensmodelle siehe WAGNER UND TAUDES (1987).

Der Einsatz der Statistik beschränkte sich hier auf die Definition vernünftiger Kennzahlen (in Zusammenarbeit mit dem Marketing) und ihrer günstigen Aufbereitung (Anordnung); erst in weiterer Folge - nachdem neue Erkenntnisse gewonnen wurden - macht man sich ein Konzept der Statistik (stochastischer Prozeß) zu Nutze. 


\subsection{Generalisierung von Resultaten mittels Metaanalysen}

Metaanalysen sind Forschungsvorhaben, in deren Verlauf eine größere Anzahl an Studien zu einem bestimmten Thema gesammelt werden, um aus der Gesamtheit an unterschiedlichen Ergebnissen allgemeingültige Aussagen ableiten zu können. Dabei werden statistische Verfahren zur Gewinnung dieser Erkenntnisse eingesetzt. Die Metaanalyse als Methode der Datenauswertung ist in konzeptueller Hinsicht von Primär- und Sekundäranalysen zu unterscheiden (vgl. GLASS, 1976). Während bei der Primäranalyse Daten zur Untersuchung vorliegen, die eigens für den vorliegenden Informationsbedarf erhoben wurden, wird bei der Sekundäranalyse mit bereits vorhandenen Daten gearbeitet. Der Zweck der sekundären Datenanalyse kann u. a. darin bestehen, neue Forschungsfragen mit bestehendem Datenmaterial zu untersuchen oder alte Hypothesen mit besseren statistischen Methoden zu überprüfen. Metaanalysen sind auf einer dritten Untersuchungsebene, einer "Meta"-Ebene, angesiedelt, da hier die Ergebnisse bereits vorliegender Studien weiteranalysiert werden. Die Durchführung einer Metaanalyse ist immer dann sinnvoll, wenn die Ergebnisse einer größeren Anzahl an Studien zu einem bestimmten Thema einzeln nicht mehr faßbar sind und einander unter Umständen sogar widersprechen.

Metaanalysen werden häufig mit Replikationsanalysen und dem Abtesten konkurrierender Modelle verwechselt. Abbildung 1 faßt daher die wesentlichen Unterschiede zwischen diesen drei Verfahren im Hinblick auf das Design und das Untersuchungsziel der jeweiligen Methode zusammen (vgl. FARLEY Und LEHMANn, 1986, S. 6).

Abbildung 1: Replikation - Konkurrierende Modelle - Metaanalyse

\begin{tabular}{|c|c|c|}
\hline Verfahren & Design & Ziel \\
\hline Replikation & $\begin{array}{l}\text { gleiches Modell, } \\
\text { gleiche Datenstruktur }\end{array}$ & $\begin{array}{l}\text { Reliabilität von } \\
\text { Resultaten }\end{array}$ \\
\hline $\begin{array}{l}\text { Abtesten konkur- } \\
\text { rierender Modelle }\end{array}$ & $\begin{array}{c}\text { verschiedene Modelle, } \\
\text { gleiche Daten }\end{array}$ & $\begin{array}{c}\text { Robustheit von Resultaten, } \\
\text { Suche nach dem besten Modell }\end{array}$ \\
\hline Metaanalyse & $\begin{array}{l}\text { verschiedene Modelle, } \\
\text { verschiedene Daten }\end{array}$ & $\begin{array}{c}\text { Generalisierung von } \\
\text { Resultaten }\end{array}$ \\
\hline
\end{tabular}

Replikationsanalysen treffen Aussagen über die Reliabilität von früher erzielten Resultaten. Zu diesem Zweck werden vergleichbare Daten auf das bestehende Modell angewendet. Beim Abtesten konkurrierender Modelle wird dasselbe Datenmaterial mit verschiedenen Modellen ausgewertet, um die Robustheit von Ergebnissen zu überprüfen oder das für den vorliegenden Anwendungsfall "beste" Modell zu identifizieren. Das Ziel von Metaanalysen besteht schließlich in der Generalisierung von Resultaten. Verschiedene Modelle mit unterschiedlichen Daten werden innerhalb einer Untersuchung zusammengefaßt, um Regelmäßigkeiten erkennen zu können.

Die Technik der Metaanalyse wird in vielen Disziplinen, etwa in der Medizin oder in den Sozial- und Wirtschaftswissenschaften angewandt. Wir beschränken uns bewußt auf 
den Fachbereich Marketing und greifen exemplarisch die Aufsätze von Assmus, FARLEY UND LEHMANN (1984) über den Einfluß der Werbung auf den Absatz und TELLIS (1988) über die Preiselastizität von selektiver Nachfrage (also der Nachfrage nach einer einzelnen Marke) heraus. Ein wichtiges Ergebnis der Untersuchung von Tellis besagt, daß die Preiselastizität ca. achtmal größer ist als die Werbeelastizität. Tellis erhielt eine mittlere Preiselastizität von -1.76, während Assmus, Farley und Lehmann über eine durchschnittliche Werbeelastizität von 0.22 berichten. Eine einprozentige Preisänderung hat also im Mittel eine achtmal größere Auswirkung auf die verkaufte Menge als eine einprozentige Änderung der Werbeausgaben. Das negative Vorzeichen der Preiselastizität spiegelt die Tatsache wider, daß eine Preiserhöhung in der Regel zu einer Verringerung der abgesetzten Stückzahl führt, während eine Erhöhung der Werbeausgaben meist einen Absatzanstieg zur Folge hat. Weitere bedeutsame Resultate der beiden Metaanalysen geben Auskunft darüber, ob einzelne Elemente der darin zusammengefaßten Studien (wie zum Beispiel Modellspezifikation, Umweltsituation, Datenart und Schätzmethode) Einflüsse auf die Höhe der jeweils erzielten Elastizitäten haben. So zeigt TELLIS (1988) beispielsweise auf, daß Marktanteilsmodelle, die keine Kennzahl bezüglich der Qualität der untersuchten Produkte beinhalten, die absolute Höhe der Preiselastizität tendenziell unterschätzen. Manager, die mit einem solchem Modell arbeiten, glauben daher, daß der Markt weniger sensibel auf Preisänderungen reagiert als dies tatsächlich der Fall ist.

Aus methodischer Sicht ist anzumerken, daß Metaanalysen meist Abhängigkeitsuntersuchungen beinhalten. Es wird erforscht, ob verschiedene Charakteristika der ausgewählten Studien einen Einfluß auf die jeweilige abhängige Variable (in den obigen Beispielen die Werbe- und Preiselastizität) ausüben. Wichtig ist es, solche Variablen als Untersuchungsgegenstand zu wählen, die über mehrere Studien hinweg vergleichbar sind, wie z. B. Gütemaße, standardisierte Koeffizienten oder Elastizitäten. Die statistische Analyse selbst wird in der Regel mittels Varianz- bzw. Kovarianzanalysen durchgeführt.

Die Wichtigkeit von Metaanalysen in der aktuellen Marketing-Forschung zeigt sich auch darin, daß seit dem Jahr 1996 ein neues, internationales Publikationsmedium, das Journal of Empirical Generalisations in Marketing Science (ein Internet-Journal) existiert, dessen Hauptaugenmerk u. a. auf der Veröffentlichung von Replikations- und Metaanalysen liegt.

\section{Der Einsatz der Statistik bei der betrieblichen Ent- scheidungsfindung am Beispiel Lauda Air}

Abschnitt 4 beschreibt ein Projekt, das vom Herbst 1996 bis zum Frühjahr 1997 vom Lehrstuhl für Marketing der Universität Wien gemeinsam mit der Lauda Air durchgeführt wurde.

Seit dem Jahr 1996 nimmt die Lauda Air in regelmäßigen Abständen Befragungen vor, um Informationen über die Zufriedenheit ihrer Kunden zu erhalten. Die Interviews erfolgen mittels standardisierter Fragebögen, die jeweils an alle Passagiere eines Fluges kurz vor der Landung ausgeteilt werden. Die Zufriedenheit wird anhand einer fünfteiligen 
Rating-Skala in den Bereichen

- Flugbegleiter (Freundlichkeit, Professionalität),

- Speisen und Getränke (Getränkeauswahl, Präsentation der Speisen, Geschmack),

- Angebot Bordverkauf,

- Unterhaltung (Videoprogramm, Musikprogramm, Angebot Tageszeitungen, Angebot Magazine, Bordmagazin) und

- Kabine (Sauberkeit, Sitzkomfort)

bewertet. Zuletzt wird der Reisende noch gebeten, ein Gesamturteil abzugeben. Mittels der erhobenen Fragebögen werden monatlich Berichte für das Management erstellt (getrennt nach Flugzeugtyp, Business \& Economy Class sowie Linie \& Charter), die über die durchschnittliche Zufriedenheit der Kunden innerhalb der untersuchten Bereiche Auskunft geben. Weiters kann mit Hilfe dieser Reporte festgestellt werden, ob sich das Service gegenüber dem Vormonat verbessert oder verschlechtert hat. Einer Aussage des zuständigen Marketing-Managers zufolge ist es eine Zielvorgabe der Geschäftsführung, daß die Lauda Air entsprechend ihres Slogans "Service is our success" sehr gute bzw. exzellente Rating-Werte erhalten sollte, um ihr Image zu bewahren bzw. zu pflegen. Ein merkliches Absinken des Servicegrades wäre mit dem Slogan nicht vereinbar und müßte zu raschen Gegenmaßnahmen führen.

Aufbauend auf den bestehenden Kundenzufriedenheitsuntersuchungen wurden weitere Verbesserungen angestrebt. Einerseits sollten zusätzlich zu den Berichten für das Management auch separate Aufstellungen für die Crewmitglieder, die Catering-Firmen und das Putzpersonal verfügbar gemacht werden, um jeden Mitarbeiter (firmenintern oder -extern) über die Zufriedenheit der Kunden im jeweiligen Bereich zu informieren. Andererseits sollte das Berichtswesen generell "verfeinert" werden. Es könnte zum Beispiel $\mathrm{zu}$ fehlerhaften Schlußfolgerungen führen, wenn die Zufriedenheitswerte eines österreichischen Catering-Betriebes gemeinsam mit den Werten eines Catering-Unternehmens aus Großbritannien innerhalb eines einzigen gemeinsamen Mittelwerts ausgewiesen werden, obwohl etwa die Zustimmung zu den Speisen der österreichischen Firma regelmäßig höher wäre. In einem solchen Fall wäre es sinnvoll, für beide Zulieferer einen getrennten Bericht zu erstellen. Der zuständige Marketing-Manager der Lauda Air stand vor dem Problem, ein geeignetes Kriterium zu entwickeln, um eine Aufspaltung der monatlichen Gesamtberichte in mehrere Teilreporte vornehmen zu können. Der Lehrstuhl für Marketing wurde beauftragt, der Unternehmensleitung bei der Gestaltung des neuen Berichtswesens behilflich zu sein. Dabei sollten gemäß einer Vorgabe des Managements statistische Methoden aus Standardsoftwarepaketen (im konkreten Fall SPSS für Windows) zur Entscheidungsunterstützung herangezogen werden.

Wir erhielten die gesammelten Kundenzufriedenheitsdaten für den Zeitraum März bis September 1996, die einem Umfang von ca. 80.000 Fragebögen entsprachen, und einigten uns auf die folgenden Analyseschritte: 
1. Zunächst wurde untersucht, ob innerhalb der fünf Bereiche des Fragebogens Unterschiede zwischen den korrespondierenden Hin- und Rückflügen bestehen.

2. In weiterer Folge wurde analysiert, ob es Differenzen zwischen den alternativen Destinationen eines Landes gibt.

3. Zuletzt wurde schließlich nachgeforscht, ob Gegensätze zwischen den angeflogenen Ländern innerhalb Europas bzw. zwischen den verschiedenen Kontinenten außerhalb Europas existieren.

Die statistische Analyse umfaßte die Durchführung von t-Tests, Varianzanalysen und verschiedenen post hoc-Tests. Folgende signifikanten Unterschiede konnten unter anderen festgestellt werden (bei einem Signifikanzniveau von $5 \%$ ):

- Speisen aus Wien werden als geschmacklich besser wahrgenommen als jene aus Barcelona, die ihrerseits wiederum besser bewertet werden als die in Lissabon gelieferte Verpflegung. Dieses Ergebnis ist insofern bemerkenswert, da die entsprechenden Zufriedenheitswerte in hohem Maße mit dem Gesamturteil korrelieren, während die Beurteilung der anderen vier Teilbereiche nur geringen Einfluß auf das Gesamturteil ausübt.

- Die Sauberkeit der Maschinen wird bei Flügen nach/von Deutschland schlechter bewertet als bei Flügen in alle anderen europäischen Länder (Großbritannien, Frankreich, Italien, Belgien, Spanien und Bulgarien).

- Im Gegensatz zu allen anderen europäischen Destinationen wird der Komfort bei Flügen nach/von Deutschland und Spanien schlechter beurteilt.

Die Ergebnisse der statistischen Untersuchungen wurden dem Management von Lauda Air vorgelegt, um nunmehr als Entscheidungsgrundlage für die Gestaltung des neuen Berichtswesens zu dienen. Mittelfristig ist es auch das Ziel des zuständigen MarketingManagers, die oben angeführten statistischen Auswertungen zu automatisieren. Beim Eintreffen von neuem Datenmaterial sollen die Signifikanztests in regelmäßigen Abständen standardmäßig durchgeführt werden, um so jederzeit aktuelle Aufschlüsse über die Zufriedenheit der Kunden von Lauda Air zu erhalten.

Bemerkenswert - aber nach unseren Erfahrungen durchaus typisch - ist an diesem Fallbeispiel die Tatsache, daß mit relativ geringem methodischem Aufwand aus bereits bestehenden Daten durch den Einsatz der Statistik wertvolle Informationen extrahiert werden können.

\section{Zusammenfassung und Ausblick}

Die vorangegangenen Abschnitte sollten einen Eindruck von der Diskrepanz zwischen der gängigen Verwendung von statistischen Verfahren in der österreichischen Markt- 
und Meinungsforschung und ihrem Potential in typischen Aufgabenstellungen des Marketing vermitteln. Aus diesem Mißverhältnis ergeben sich einige Folgerungen für die Vermittlung des Faches Statistik im Rahmen der universitären Ausbildung für die Zielgruppe "künftige (Marketing-)Manager". Vorrangig muß das grundlegende Verständnis für das Fach gefördert und die nutzbringende Anwendung auf Probleme der Praxis demonstriert werden. Dabei gilt es die Grenzen aufzuzeigen, die insbesondere auf Grund von (fehlerbehafteten) empirischen Daten abgesteckt werden. Ein derartiges Wissen wird den Nutzer statistischer Analysen vor voreiligen Schlüssen und in weiterer Folge auch vor Enttäuschung oder Frustration bewahren. Andererseits sollten applikationsbezogene Kenntnisse helfen, die Stärken und die potentiellen Einsatzmöglichkeiten statistischer Methoden stets vor Augen behalten zu können.

Auch für Forschungsaktivitäten an der Schnittstelle zwischen Statistik und Marketing ergeben sich einige Empfehlungen. Häufig müssen hier Probleme bearbeitet werden, die durch einen geringen Stichprobenumfang und eine relativ schlechte Datenqualität gekennzeichnet sind, bei denen regelmäßig Grundannahmen statistischer Verfahren verletzt werden, bei denen subjektive Informationen (beispielsweise von Managern) berücksichtigt werden sollten, die aber dennoch - möglicherweise unter Verzicht auf große Genauigkeitsanforderungen - einer robusten Lösung zugeführt werden sollen. Statistiker und Marktforscher sind aufgerufen, gemeinsam die Eigenschaften bekannter statistischer Methoden unter den oben beschriebenen Bedingungen auszuloten sowie eigens auf Marketingprobleme konzipierte Verfahren zu entwickeln.

Zusammenfassend gilt für Lehre und Forschung ganz im Sinne John Tukeys:

"Far better an approximate answer to the right question, which is often vague, than an exact answer to the wrong question, which can always be precise."

\section{Literatur}

Assmus, G., Farley, J.U., Lehmann, D.R. (1984). How Advertising Affects Sales: Meta-Analysis of Econometric Results. Journal of Marketing Research 21, 6574.

Bass, F.M., Leone, R.P. (1983). Temporal Aggregation, the Data Interval Bias, and Empirical Estimation of Bimonthly Relations from Annual Data. Management Science 29, 1, 1-11.

Dewdney, A.K. (1994). 200 Prozent von nichts. Basel: Birkhäuser.

Ehrenberg, A.S.C. (1988). Repeat-Buying: Facts, Theory and Applications. New Edition. London: Charles Griffin.

Farley, J.U., Lehmann, D.R. (1986). Meta-Analysis in Marketing: Generalisation of Response Models. Lexington: Lexington Books.

GLAss, G.V. (1976). Primary, Secondary, and Meta-Analysis of Research. Educational Research 5, 3-8. 
KRÄMER, W. (1995). So lügt man mit Statistik. Frankfurt: Campus.

SACHS, A. (1997). Prozent-Protze. Bestseller 3, 50-51.

Tellis, G.J. (1988). The Price Elasticity of Selective Demand: A Meta-Analysis of Econometric Models of Sales. Journal of Marketing Research 25, 331-341.

Wagner, U., TAudes, A. (1987). Stochastic Models of Consumer Behaviour. European Journal of Operational Research 29, 1-23.

Adresse der Autoren:

o.Univ.-Prof. Dr. Udo Wagner

Dr. Heribert Reisinger

Institut für Betriebswirtschaftslehre,

Lehrstuhl für Marketing, Universität Wien

Brünner Straße 72, A-1210 Wien

E-Mail: reisinger@marketing.bwl .univie.ac .at 